\title{
Eco-etología básica del caracol rosa Strombus gigas (Mesogastropoda: Strombidae), en Xel-Há, Yucatán, Caribe mexicano
}

\author{
Mariana Noguez Núñez ${ }^{1,2} \&$ Dalila Aldana Aranda ${ }^{2}$ \\ 1. Posgrado en Ciencias del Mar y Limnología, Universidad Nacional Autónoma de México (ICMyL-UNAM). Puerto \\ de Abrigo s/n, Sisal. C.P. 97351, Hunucmá, Yucatán, México; biologa_mariana@yahoo.com \\ 2. Laboratorio de Biología y Cultivo de Moluscos. Centro de Investigación y de Estudios Avanzados del Instituto \\ Politécnico Nacional, Unidad Mérida (CINVESTAV-IPN). Carretera Antigua a Progreso, Km. 6, A.P. 73 Cordemex, \\ C.P. 97310, Mérida, Yucatán, México. Tel. +52(999) 9429400 Ext. 2538; daldana@mda.cinvestav.mx.
}

Received 15-VIII-2013 Corrected 12-III-2014 Accepted 01-IV-2014

\begin{abstract}
Basic eco-ethology of the queen conch, Strombus gigas (Mesogastropoda: Strombidae) in XelHá, Yucatán, Mexican Caribbean. The queen conch Strombus gigas is an important fishery in the Caribbean, whose populations are currently overexploited. Since the decade of 1980 there have been several studies on aquaculture, resource management and area rehabilitation. However, little is known about its behavior in a natural environment and the influence of environmental parameters. Monthly surveys, from January to November 2012 were conducted in in Xel Ha, to observe and quantify six behaviors of $S$. gigas: rest, feeding, movement, burying, copulation and spawning. The observations were made every hour from $8 \mathrm{~h}$ to $17 \mathrm{~h}$ by free diving through three transects with three replicates each. Each behavior was observed 90 times each month. Salinity, temperature and dissolved oxygen were registered at the bottom. We found the highest number of snails at rest in July and the lowest in March and September. Feeding and movements had a peak in August. Most buried in October and November. Copulation was first observed on March with a peak in June and July to October's spawning. In daytime observations restingt had a peak at $8 \mathrm{~h}$; feeding and moving at 12 to $17 \mathrm{~h}$. Copulation and spawning did not have a clear pattern. Variations between months and hours (resting, feeding, moving and buried) were significant $(\mathrm{p}<0.05)$. Resting correlated with temperature and being buried with oxygen level ( $\mathrm{r}=-$ $0.5803 ; \mathrm{p}=0.0536$ ). Feeding and moving correlated with temperature and salinity. These results should be useful for the conservation, restoration and aquaculture programs. Rev. Biol. Trop. 62 (Suppl. 3): 215-222. Epub 2014 September 01 .
\end{abstract}

Key words: Behavior, conservation, feeding, move, spawning, queen conch.

El caracol rosa cuyo nombre científico actual es Strombus gigas (Linnaeus, 1758) es un gasterópodo herbívoro que llega a medir $30 \mathrm{~cm}$ de longitud sifonal y a pesar $5 \mathrm{~kg}$ a su madurez sexual entre los 5-6 años. Se le encuentra en aguas territoriales de 36 países y territorios del Mar Caribe (Davis, 2005), sur de Florida, Centroamérica, noreste de Brasil, Bahamas y Bermudas. Habita en lechos arenosos, en cama de pastos o en escombros de coral (Brownell \& Stevely, 1981). S. gigas es vulnerable a la sobrepesca debido a su lento crecimiento, formación de grandes aglomeraciones en aguas profundas, tardía maduración y tendencia a desovar en aguas someras. Además, tiene importancia comercial y artesanal en el Caribe siendo la segunda pesquería más importante después de la langosta espinosa (Davis, 2005). En los 1970's, la densidad de adultos de $S$. gigas iba de cientos a miles de individuos por hectárea, actualmente la densidad se encuentra por debajo de los 100 individuos por hectárea en la mayoría de las regiones del Caribe. La disminución de las poblaciones del caracol rosa hizo que a partir de 1992 fuera enlistada en el apéndice II de CITES, donde se encuentran especies amenazadas o que necesitan estricta regulación (CITES, 2003). Se han 
realizado estudios sobre su cultivo, ecología y manejo pesquero, dentro de éste último punto, se han establecido vedas temporales y permanentes (Davis, 2005), así como áreas marinas protegidas y cuotas de captura (Aldana \& Brulé, 1994). La efectividad de las áreas protegidas depende de la identificación de zonas de desove así como áreas de crianza, sin embargo, en muchos países los esfuerzos de conservación son pobres, y la sobrepesca, así como la pesca ilegal sigue ocurriendo (CITES, 2003; FAO, 2007). Para complementar los estudios que se han hecho para la conservación de esta especie es necesario conocer algunos de sus comportamientos como: reposo, movimiento, alimentación, enterrados, cópula y desove, que son los más reportados en la literatura, sin embargo, éstos estudios previos no presentan un patrón de comportamiento a través del tiempo, ni los relacionan con los parámetros ambientales, por lo que el principal objetivo de éste estudio fue conocer el patrón de comportamiento de $S$. gigas en diferentes escalas de observación temporal (diaria y anual) y su relación con los parámetros ambientales. Los resultados obtenidos en éste estudio serán de importancia para el cultivo de la especie, ya que, al conocer los periodos diarios y/o anuales de su comportamiento se puede contar con las instalaciones adecuadas y optimizar la administración del alimento.

\section{MATERIALES Y MÉTODOS}

Área de estudio y muestreo: Xel-Há se ubica en la costa de la Península de Yucatán en el Caribe mexicano, entre los $20^{\circ} 20^{\prime}$ latitud $\mathrm{N}$ y $\operatorname{los} 87^{\circ} 21^{\prime}$ longitud $\mathrm{W}$. La caleta tiene una superficie de $0.3 \mathrm{~km}^{2}$ y presenta tres apéndices (Aldana et al., 2005). La principal característica es la mezcla de agua dulce y salobre, tiene una profundidad de 2 a $4 \mathrm{~m}$ y fondos principalmente arenosos con parches de pastos marinos. Se eligió Xel-Há por ser una área marina protegida, donde la remoción de flora y fauna está prohibida; además es considerada un santuario para la conservación del caracol rosa en la Rivera Maya Mexicana (Peel et al.,
2010), ya que sostiene una densidad de juveniles y adultos por arriba de lo que recomienda Stoner (2002).

El sitio que se eligió para monitorear el comportamiento de los caracoles adultos dentro de la Caleta de Xel Há fue "Bocana", que es la zona que comunica al mar y presenta un sustrato arenoso-medio con parches de pastos marinos. Los comportamientos cuantificados en este estudio fueron: reposo (organismos donde se observa la concha sobre el sedimento sin ningún desplazamiento y la probóscide se encuentra retraída), alimentación (organismos con la probóscide fuera de la concha realizando escaneo sobre el fondo o los pastos marinos), movimiento (organismos que por medio de pequeños saltos continuos se desplazan de un punto a otro), enterrado (organismos que únicamente muestran las espinas, el canal y la muesca sifonal de la concha y el resto está cubierto por sedimento), cópula (dos caracoles alineados donde la parte delantera de la concha del macho está cubriendo la parte posterior de la hembra) y desove (organismos donde se observa una madeja parecida al estambre, de color blanquecino saliendo de la concha del caracol). Mensualmente de enero a noviembre de 2012 se recorrieron por triplicado tres transectos de $100 \mathrm{~m}$ de longitud por $2 \mathrm{~m}$ de ancho, anotando en cada recorrido el número de caracoles en cada uno de los comportamientos señalados. Las observaciones fueron realizadas cada hora por medio de buceo libre de 8 a las $17 \mathrm{~h}$, con un número alto de observaciones $(n=10800)$. Simultáneamente, se registraron temperatura, salinidad y oxígeno disuelto de fondo con un YSI 85.

Análisis estadísticos. Se realizaron gráficas de barras con desviación estándar por comportamiento a través de las horas y los meses del año (INFOSTAT versión 1.1, 2002). Así mismo, se elaboraron histogramas con la finalidad de conocer si los datos eran normales, en el caso de no ser normales se realizó una transformación utilizando la fórmula $\operatorname{Ln}(\mathrm{x}+1)$. Se realizaron ANOVAS de una vía entre las categorías de comportamiento cuantificadas y 
el tiempo (horas y meses), con la finalidad de conocer si existen diferencias del comportamiento a través del tiempo, y en caso de existir diferencias significativas se aplicó un análisis post hoc (Tukey HSD).

Para analizar el posible efecto de los parámetros ambientales se elaboraron gráficas de dispersión con línea de tendencia, y se calculó el valor de la correlación de Pearson así como su significancia; se utilizaron los promedios de temperatura, salinidad y oxígeno disuelto de todos los meses así como los promedios de los comportamientos dominantes (mayor número de organismos y observados durante todo el año) utilizando el programa STATISTICA 8, 2006.

\section{RESULTADOS}

Todos los comportamientos del caracol rosa presentaron diferencias significativas a través de los meses, sin embargo, en un ciclo diurno el comportamiento reproductivo no presenta variación. En relación a los parámetros ambientales, la temperatura es la que influye más en el comportamiento del caracol que las otras variables.

\section{Comportamiento mensual del caracol} rosa de $S$. gigas: El reposo presentó diferencias significativas a través de los meses (ANOVA $\mathrm{p}<0.05$ ), con el mayor número de organismos en julio y el menor número en marzo y septiembre (Fig. 1a). La alimentación mostró diferencias significativas entre meses (ANOVA $\mathrm{p}<0.05$ ), con un pico entre febrerooctubre del $35 \%$ en promedio y valores bajos en enero y noviembre (Fig. 1b). El movimiento presentó diferencias significativas entre meses (ANOVA $\mathrm{p}<0.05$ ), agosto y septiembre con el mayor número de organismos (16\%) y julio y noviembre con el menor número de organismos (4\%) (Fig.1c). En octubre y noviembre se observó el mayor número de organismos enterrados y en mayo el menor número de caracoles con este comportamiento, habiendo diferencias significativas entre meses (ANOVA $\mathrm{p}<0.05$ ) (Fig. 1d). La cópula se empezó a observar en marzo, con un pico en junio y julio presentando diferencias significativas (ANOVA $\mathrm{p}<0.05$ ) de estos meses con el resto del año (Fig. 1e). El desove se observó de julio a octubre. En julio se tuvo el pico de desove presentando diferencias significativas (ANOVA p $<0.05$ ) (Fig. 1f).

Comportamiento diurno del caracol $S$. gigas: Los caracoles presentaron en sus actividades un ciclo diurno. Por lo que se refiere al reposo, este fue alto a las $8 \mathrm{~h}$, donde $70 \%$ de la población de caracoles se encontró en éste comportamiento. El mínimo se observó a las $14 \mathrm{~h}(33 \%)$, habiendo diferencias significativas entre horas (Fig. 2a). La alimentación aumentó de manera gradual de las $8 \mathrm{~h}$ a las $14 \mathrm{~h}$, manteniéndose con valores altos hasta las $17 \mathrm{~h}$ (promedio $39-41 \%$ de la población en alimentación). La prueba de ANOVA $(\mathrm{p}<0.05)$ mostró diferencias significativas en la alimentación entre horas (Fig. 2b). Los caracoles presentaron un patrón de movimiento diurno, no teniendo variación de las 10-17h (10\%), sin embargo, en las primeras horas de la mañana la motilidad fue muy reducida $(<5 \%)$. La prueba de ANOVA $(\mathrm{p}<0.05)$ mostró diferencias significativas del movimiento entre horas (Fig.2c). Los caracoles se enterraron más por la mañana (8-9h) que en la tarde, habiendo diferencia significativa entre horas (Fig. 2d). La cópula así como el desove no presentaron diferencias significativas a través de las horas (ANOVA p>0.05).

Interacción entre comportamiento y variables ambientales: Se realizaron correlaciones de Pearson entre los comportamientos que se cuantificaron de $S$. gigas (reposo, movimiento, alimentación y enterrados) a lo largo de un año de muestreo con observaciones diurnas y mensuales y los parámetros temperatura, salinidad y oxígeno disuelto. El reposo y la alimentación presentaron una correlación significativa y alta con la temperatura ( $\mathrm{r} 0.70558$ $[\mathrm{p}=0.0171]$ y $\mathrm{r} 0.66304[\mathrm{p}=0.0503]$ respectivamente), además la alimentación presentó una correlación significativa con la salinidad $(\mathrm{r}=0.6305 \quad \mathrm{p}=0.0216]) \quad($ Figs. 3a y 3b). El movimiento también presentó una correlación 

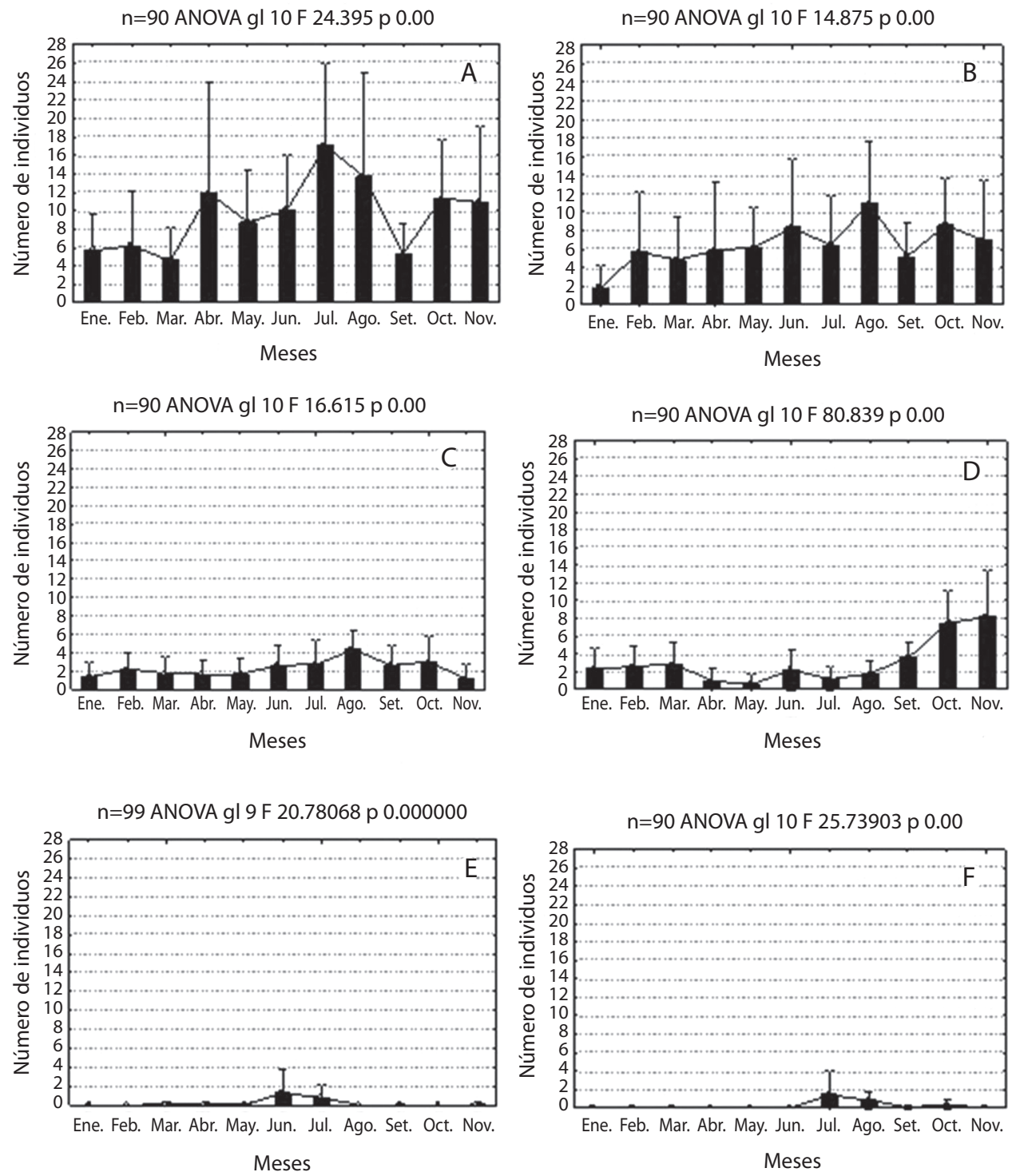

Fig. 1. Variaciones mensuales del comportamiento de $S$. gigas: a) reposo, b) alimentación, c) movimiento, d) enterrados, e) cópula y f) Desove. *diferencia significativa, $\mathrm{n}=90$.

Fig. 1. Monthly behavior of queen conch $S$. gigas: a) resting, b) feeding, c) moving, d) buried, e) copulating, f) spawning. *Significant difference, $\mathrm{n}=90$.

significativa, alta y directa con la temperatura (r 0.73647 [p=0.0022]) (Fig. 3c). El porcentaje de caracoles enterrados presentó una correlación negativa y significativa con el oxígeno disuelto ( $\mathrm{r}-0.5803$ [p=0.0536]) (Fig. 3d).

\section{DISCUSIÓN}

A lo largo del año los organismos de $S$. gigas presentaron variaciones significativas en sus comportamientos. Se observó actividad 
$\mathrm{n}=99$ ANOVA gl $9 \mathrm{~F} 11.258 \mathrm{p} 0.000000$

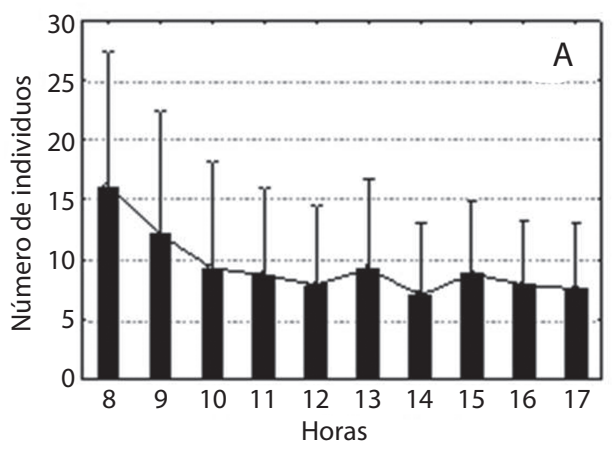

$\mathrm{n}=99$ ANOVA gl $9 \mathrm{~F} 19.759 \mathrm{p} 0.00$

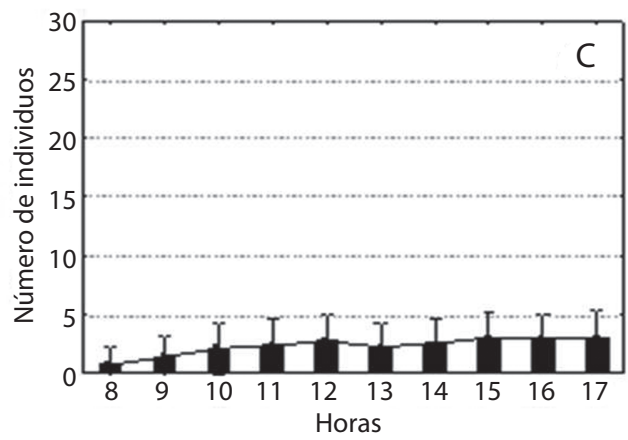

$\mathrm{n}=99$ ANOVA gl $9 \mathrm{~F} 19.759$ p 0.00

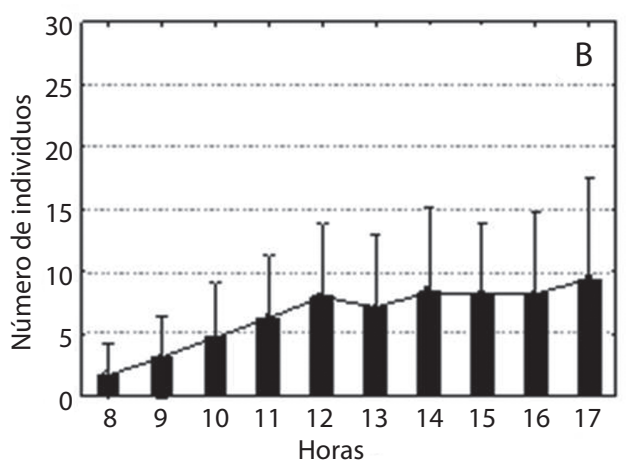

$\mathrm{n}=99$ ANOVA gl $9 \mathrm{~F} 6.593 \mathrm{p} 0.000000$

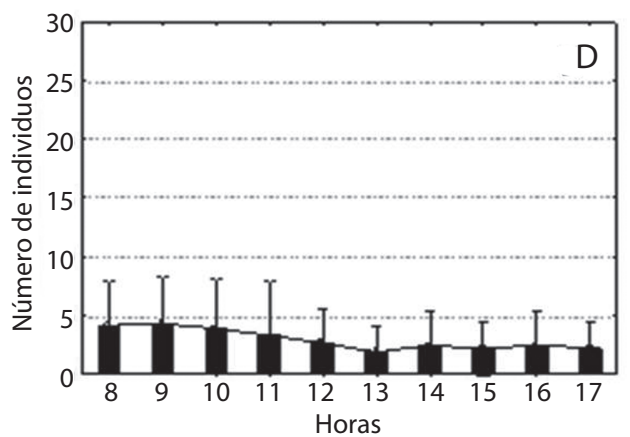

Fig. 2. Variaciones diurnas del comportamiento de S. gigas: a) reposo, b) alimentación, c) movimiento, d) enterrados. *diferencia significativa, $\mathrm{n}=99$.

Fig. 2. Diurnal behavior of queen conch $S$. gigas: a) resting, b) feeding, c) moving, d) buried. * Significant difference, $\mathrm{n}=99$.

reproductiva (cópula y desove) de marzo a octubre, resultados que coinciden con lo reportado por Aldana Aranda et al. (2005; 2006). La cópula empezó a observarse en marzo con un pico en junio, coincidiendo a lo reportado para el Arrecife Alacranes (Pérez \& Aldana, 2003) y lo observado en Turk y Caicos, por Davis, Hesse y Hodgkins (1987), con esto queda demostrado que el caracol rosa presenta un periodo de reproducción, lo cual debe tomarse en cuanta a la hora de establecer los periodos de veda. Randall (1964) observó que los caracoles se entierran hasta la base de la espina principalmente en invierno o por efecto de fuerte marea y Hesse (1979) observó que cuando el mar está tempestuoso por efecto de vientos del este y del norte los caracoles se entierran (noviembre a febrero). Jesús Navarrete (comm pers) señala que el caracol puede enterrarse por periodos prolongados en sus primeras etapas de desarrollo o cuando las condiciones ambientales son adversas. En éste estudio el mayor número de caracoles enterrados se encontró de octubrefebrero, que corresponde a la época de nortes, coincidiendo con lo reportado por los autores antes señalados. El movimiento tuvo un pico en agosto, coincidiendo con lo que mencionan Stoner y Sandt (1992), quienes señalan que la temporada de migración de esta especie está generalmente asociada al desove, donde los caracoles migran de aguas profundas a someras para la reproducción (Randall, 1964). Sin embargo, Aldana (2006) señala que $S$. gigas tiene una migración reproductiva y que ésta se encuentra influenciada por la granulometría del sedimento, ( $S$. gigas prefiere un diámetro de partículas de arenas medias para el desove) y no solo por la batimetría o el efecto Alee 

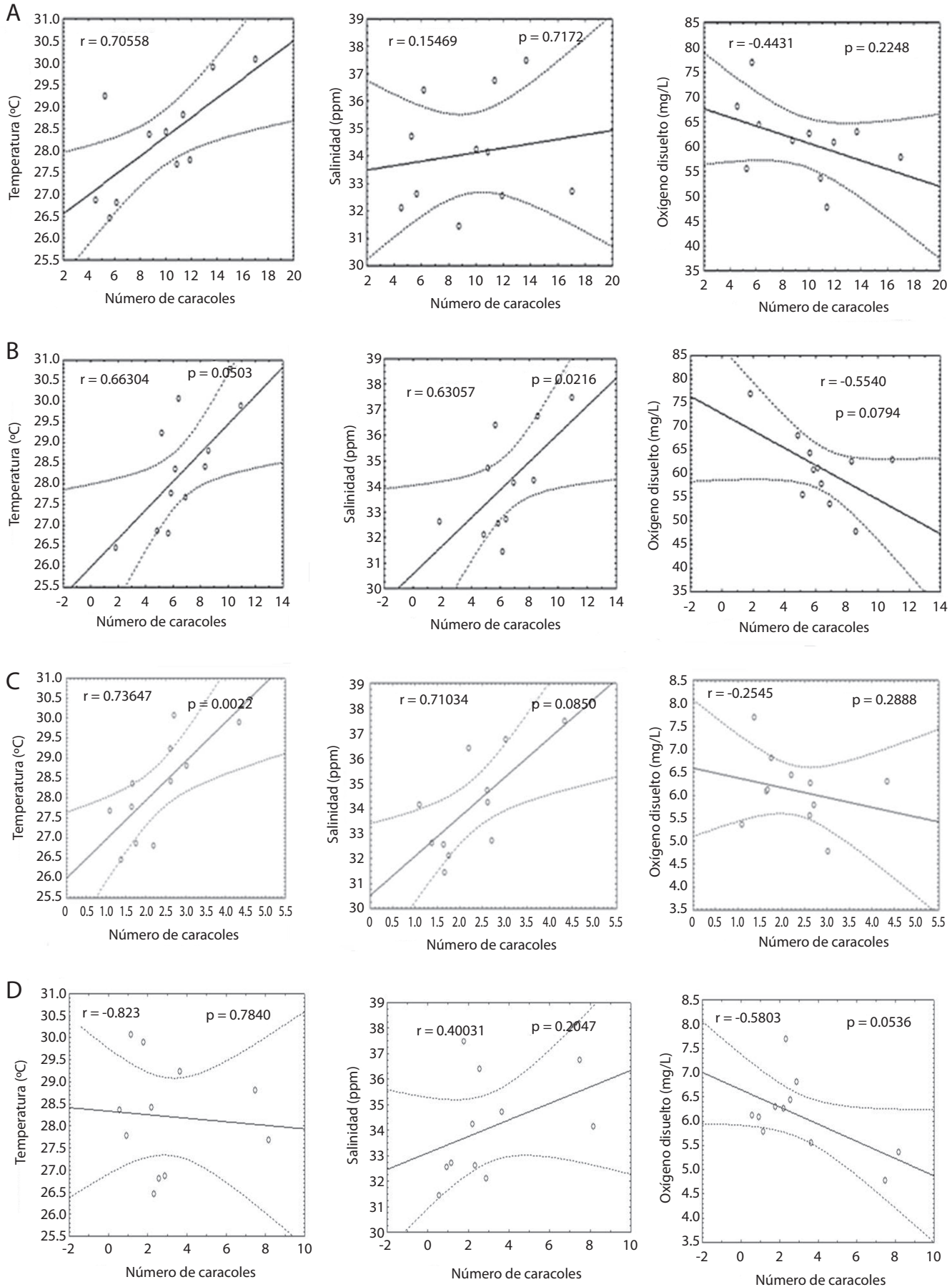

Fig. 3. Correlación de los comportamientos en S. gigas de reposo (a), alimentación (b), movimiento (c), enterrados (d) con la temperatura, salinidad y con oxígeno disuelto.

Fig. 3. Correlation of behavior of $S$. gigas (a), resting, b) feeding, c) moving, d) buried with temperature, salinity and dissolved oxygen. 
descrito por Stoner y Ray-Culp (2000). Entre la alimentación y el movimiento existe una relación directa, por lo que se afirma que el caracol requiere desplazarse para alimentarse, presentando un ciclo diurno en su alimentación con dos picos de ingesta: $12-17 \mathrm{~h}$ y otro nocturno (22h) ésta observación concuerda con lo reportado por De Santis (1982) que observa un aumento de la actividad alimentaría en paralelo con un incremento de la motilidad. Randall (1964) reportó que $S$. gigas se alimenta todo el día sin precisar la metodología empleada. De Santis (1982) en el archipiélago de los Roques Venezuela, observa cuatro picos en la alimentación: $8 \mathrm{am}, 12 \mathrm{pm}, 2 \mathrm{pm}$ y 4-5pm. En éste estudio se encontró que la alimentación y el movimiento presentan un pico entre $12-5 \mathrm{pm}$, señalando que las curvas de inactividad (reposo/enterrados) son imagen especular de las curvas de actividad (movimiento/alimentación).

Orr y Berg (1987) reportan que el movimiento de $S$. gigas depende de la temperatura y que éste es mayor durante los meses cálidos; lo cual coincide con lo observado en éste trabajo, con correlación positiva alta entre temperatura con alimentación y movimiento. El conocimiento del patrón del comportamiento de $S$. gigas obtenido en este trabajo es el primero en que se realiza para una serie de tiempo con observaciones temporales en un ciclo diurno y mensual durante todo un año. Es un aporte además original, dónde esta investigación aporta información valiosa al conocimiento de la ecología y la etología de $S$. gigas, siendo esta útil para los programas de conservación y restauración de poblaciones en áreas marinas protegidas y para su cultivo, en las fases de engorde de juveniles y maduración reproductiva de adultos en sistemas de encierros.

\section{AGRADECIMIENTOS}

\section{Al proyecto INNOVATEC-CONACYT} No. 183724 "Sistemas de información geográfica para la conservación del patrimonio del Caribe y el desarrollo sustentable del Parque Xel-Há”. A CONACYT por la Beca de maestría 2011-2013, No.: 271214. A la infraestructura del laboratorio de Biología y cultivo de moluscos CINVESTAV IPN Unidad Mérida y a su equipo por el apoyo en los muestreos (Manuel Sánchez Crespo, Luis Alfonso Ascencio Aguirre, José Francisco Chávez Villegas, Nuria Estrada Saldívar, Irma Pérez García, Wendy González López, Alejandro Aldana Moreno y Francisco Gameros Escoto). Al Parque Xel-Há por su apoyo logístico y operacional y facilidades brindadas durante los muestreos.

\section{RESUMEN}

El caracol rosa Strombus gigas tiene importancia pesquera en el Caribe, cuyas poblaciones se encuentran sobreexplotadas. Desde la década de 1980 se han desarrollado estudios de acuacultura, manejo del recurso y rehabilitación de áreas con la finalidad de proteger e incrementar sus poblaciones. Sin embargo, poco se sabe sobre el comportamiento del caracol rosa en su medio natural y la influencia de los parámetros ambientales. Mensualmente de enero a noviembre de 2012 en la caleta de Xel Há se observaron y cuantificaron seis comportamientos de $S$. gigas: reposo, alimentación, movimiento, cópula, enterrados y desove. Las observaciones se realizaron cada hora de $8 \mathrm{~h}$ a $17 \mathrm{~h}$ por medio de buceo libre en tres transectos con tres repeticiones cada uno. Cada comportamiento fue observado 90 veces cada mes. Se registraron datos de salinidad, temperatura y oxígeno disuelto en el fondo. El comportamiento anual de $S$. gigas presentó el mayor número de caracoles en reposo en julio. La alimentación y el movimiento tuvieron un máximo en agosto. La mayoría de caracoles enterrados se encontró en octubre y noviembre. La cópula comenzó a observarse en marzo con un máximo en junio y el desove de julio a octubre. En las observaciones diurnas e independientemente de los meses, el reposo tuvo un pico a las $8 \mathrm{~h}$, alimentación a las 12 y movimiento a las $17 \mathrm{~h}$. La cópula y el desove no presentaron un patrón en su comportamiento. Existieron variaciones significativas entre meses y horas (reposo, alimentación, movimiento y enterrado) $(\mathrm{p}<0.05)$. El reposo se correlacionó con la temperatura y los enterrados con el oxígeno $(\mathrm{r}=-0.5803 ; \mathrm{p}=0.0536)$. La alimentación con temperatura, salinidad y oxígeno. El movimiento con temperatura y salinidad. Estos resultados son útiles para programas de conservación, restauración y cultivo de la especie.

Palabras clave: caracol rosa, comportamiento, conservación, alimentación, desove, movimiento.

\section{REFERENCIAS}

Aldana, D., \& Brulé, T. (1994). Estado Actual de la pesquería, del cultivo y programa de investigación del 
caracol Strombus gigas en México. En Appeldoorn, R. S., \& Rodríguez, Q. B. (eds). Queen Conch Biology, Fisheries and Mariculture. Caracas, Venezuela: Fundación Científica Los Roques.

Aldana, D. (2006). Overview del patrón reproductivo del caracol Strombus gigas para diferentes localidades del Caribe. 57th Gulf and Caribbean Fisheries Institutes, 771-790.

Aldana, D., Sánchez, M., Reynaga, P., Patiño, V., George, A., \& Baqueiro, E. R. (2005). Crecimiento y temporada reproductiva del caracol rosado Strombus gigas en el Parque Xel-Há, México. 56th Gulf and Caribbean Fisheries Institutes, 742-754.

Brownell, W. N., \& Stevely, J. M. (1981). The biology, fisheries, and management of the queen conch, Strombus gigas. Marine Fisheries Review, 43(07), 1-12.

CITES-Convention of International Trade in Endangered Species of Wild Fauna and Flora. (2003). Review of significant trade in specimens of Appendix II species. 19th Meeting of the Animals Committee, Geneva Switzerland.

Davis, M. (2005). Species Profile Queen conch Strombus gigas. SRAC Publication No. 7203.

Davis, M., Hesse C., \& Hodgkins, G. (1987). Commercial hatchery produced queen conch, Strombus gigas, seed for the research and grow-out market. Proceedings of the Gulf and Caribbean Fisheries Institutes, 38, 326-335.

De Santis, C. (1982). Algunos aspectos de la ecología trófica del Botuto (Strombus gigas) con miras a su cultivo en el Archipiélago de los Roques, Venezuela. Tesis de Licenciatura. Universidad Central de Venezuela. Caracas, Venezuela.

FAO. (2007). Report on the Regional Workshop on the monitoring and management of Queen Conch, Strombus gigas. Kingston, Jamaica: FAO Fisheries Report No. 832.

Hesse, K. O. (1979) Movement and migration of the queen conch, Strombus gigas, in Turks and Caicos Islands. Bulletin of Marine Science, 29(3), 303-311.

Orr, K. S., \& Berg, C. J. Jr. (1987). The Queen Conch. Miami, USA: Finney Company Incorporated.

Peel, J. R., Saenz, R., May, E., Montero, J., \& AldanaAranda, D. (2010). Importance of a Marine Protected Area in the Mexican Caribbean on the Conservation of the Endangered Species of Queen Conch, Strombus gigas. GCFI Book of Abstracts, 63, 39.

Perez, M., \& Aldana, D. (2003). Actividad reproductiva de Strombus gigas (Mesogasteropoda: Strombidae) en diferentes hábitats del Arrecife Alacranes, Yucatán. Revista de Biología Tropical Suplemento, 4, 119-126.

Randall, J. E. (1964). Contributions to the biology of the "queen conch" Strombus gigas. Bulletin of Marine Science of the Gulf and Caribbean, 14, 246-295.

Stoner, A. W., \& Sandt, V. J. (1992). Population structure, seasonal movements and feeding of queen conch, Strombus gigas in deep-water habitats of the Bahamas. Bulletin of Marine Science, 51(3), 287-300.

Stoner, A. W., \& Ray-Culp, M. (2000). Evidence for Allee effects in an over-harvested marine gastropod: density-dependent mating and egg production. Marine Ecology Progress Series, 202, 297-302. 